\title{
CHAIN SEQUENCES AND ORTHOGONAL POLYNOMIALS
}

\author{
BY
}

T. S. CHIHARA

1. Introduction. An important characterization of real orthogonal polynomials is the classical three term recurrence formula,

$$
\begin{aligned}
& P_{n}(x)=\left(x-c_{n}\right) P_{n-1}(x)-\lambda_{n} P_{n-2}(x), \\
& P_{-1}(x)=0, \quad P_{0}(x)=1, \quad(n=1,2,3, \cdots) . \\
& c_{n} \text { real, } \quad \lambda_{n+1}>0 \quad
\end{aligned}
$$

More precisely, let $\left\{P_{n}(x)\right\}$ be a sequence of polynomials, $P_{n}(x)$ of degree $n(n=0,1,2, \cdots)$, standardized by the condition that the leading coefficient of $P_{n}(x)$ is unity. Then a necessary and sufficient condition that the $P_{n}(x)$ satisfy an orthogonality relation of the form

$$
\int_{-\infty}^{\infty} P_{m}(x) P_{n}(x) d \psi(x)=0 \quad(m \neq n)
$$

where $\psi$ is a distribution (bounded, nondecreasing) function with an infinite spectrum (set of points of increase) is that (1.1) holds for some $\left\{c_{n}\right\},\left\{\lambda_{n}\right\}$ (for example, see $[9$, p. $41 ; 3]$ ). The $P_{n}(x)$ are the denominators of the $n$th convergents of the "associated" continued fraction

$$
K(z)=\frac{\lambda_{1} \mid}{\mid z-c_{1}}-\frac{\lambda_{2} \mid}{\mid z-c_{2}}-\frac{\lambda_{3} \mid}{\mid z-c_{3}}-\cdots
$$

and the above results also follow from the classical theory of continued fractions (for example, see [10] and the remarks in [5]).

In view of this, it seems natural to attempt a study of the properties of the polynomials, $P_{n}(x)$, as determined by the coefficients, $c_{n}$ and $\lambda_{n}$. One approach to such an investigation is of course through the study of the convergence of the continued fraction (1.3). In this paper, however, we shall avoid direct reference to the theory of continued fractions although we shall make fairly extensive use of the results from the problem of moments. Instead, our investigation centers upon the "true" interval of orthogonality of $\left\{P_{n}(x)\right\}$. [By the "true" interval of orthogonality, we mean, following Shohat (see $[5 ; 6$, p. 113]), the smallest interval which contains in its interior all zeros of all $P_{n}(x)$.] In this connection, the "chain sequences" of Wall [10] arise naturally and play a fundamental role. Thus our study is largely "arithmetical" in character.

Throughout this paper, "orthogonal polynomials" means polynomials

Presented to the Society, February 18, 1960; received by the editors July 31, 1961. 
orthogonal with respect to a distribution with an infinite spectrum.

2. Some preliminary relations. Given (1.1), assume that the "true" interval of orthogonality of $\left\{P_{n}(x)\right\}$ is $(a, b) \subset(0, \infty)$. Then there exists a distribution, $d \psi(x)$, whose spectrum is a subset of $[a, b]$ with respect to which the $P_{n}(x)$ are orthogonal. Let $Q_{n}(x)$ denote the unique $n$th degree polynomials with leading coefficients unity which are orthogonal on $(a, b)$ with respect to the distribution, $x d \psi(x)$. Then there are real constants, $d_{n}$ and $\nu_{n+1}>0(n \geqq 1)$, such that

$$
\begin{aligned}
Q_{n}(x) & =\left(x-d_{n}\right) Q_{n-1}(x)-\nu_{n} Q_{n-2}(x) \quad(n=1,2, \cdots), \\
Q_{-1}(x) & =0, \quad \nu_{1} \text { arbitrary. }
\end{aligned}
$$

Next define polynomials, $R_{n}(x)$, by

$$
\begin{aligned}
R_{2 n}(x) & =P_{n}\left(x^{2}\right), \\
R_{2 n+1}(x) & =x Q_{n}\left(x^{2}\right),
\end{aligned} \quad(n=0,1,2, \cdots) .
$$

Then it is readily verified from the orthogonality properties of the $P_{n}(x)$ and $Q_{n}(x)$ that the $R_{n}(x)$ are orthogonal on $\left(-b^{1 / 2}, b^{1 / 2}\right)$ with respect to the distribution

$$
d \phi(x)=(\operatorname{sgn} x) d \psi\left(x^{2}\right), \quad-b^{1 / 2} \leqq x \leqq b^{1 / 2} .
$$

Thus there exist constants, $\gamma_{n}>0(n \geqq 2)$, such that

$$
\begin{aligned}
R_{n}(x) & =x R_{n-1}(x)-\gamma_{n} R_{n-2}(x) \quad(n=1,2, \cdots), \\
R_{-1}(x) & =0, \quad \gamma_{1}=0 \text { (for later convenience). }
\end{aligned}
$$

Conversely, given the $R_{n}(x)$ satisfying (2.3), we obtain the $P_{n}(x)$ and $Q_{n}(x)$ satisfying (1.1) and (2.1), respectively, by means of (2.2). Moreover, we can easily derive explicit relations among the coefficients in (1.1), (2.1), and (2.3) as follows.

Write (2.3) with $n$ replaced by $2 n$ and use (2.2) to obtain

$$
P_{n}(x)=x Q_{n-1}(x)-\gamma_{2 n} P_{n-1}(x) \quad(n=1,2, \cdots) .
$$

Similarly, replace $n$ by $2 n+1$ in (2.3) and use (2.2) to obtain

$$
Q_{n}(x)=P_{n}(x)-\gamma_{2 n+1} Q_{n-1}(x) \quad(n=0,1, \cdots) .
$$

Now eliminate $P_{n}(x)$ from (2.5) by means of (2.4). Then use (2.5) with $n$ replaced by $n-1$ to eliminate $P_{n-1}(x)$ and obtain

$$
Q_{n}(x)=\left(x-\gamma_{2 n}-\gamma_{2 n+1}\right) Q_{n-1}(x)-\gamma_{2 n-1} \gamma_{2 n} Q_{n-2}(x) \quad(n=1,2, \cdots) .
$$

In the same way, we also obtain

$$
P_{n}(x)=\left(x-\gamma_{2 n-1}-\gamma_{2 n}\right) P_{n-1}(x)-\gamma_{2 n-2} \gamma_{2 n-1} P_{n-2}(x) \quad(n=1,2, \cdots) .
$$

Comparison of the last two relations with (1.1) and (2.1) yields 


$$
\begin{array}{lr}
c_{n}=\gamma_{2 n-1}+\gamma_{2 n}, \quad \lambda_{n+1}=\gamma_{2 n} \gamma_{2 n+1} & \left(\gamma_{1}=0\right), \\
& (n=1,2, \cdots) .
\end{array}
$$

The relations (2.6) and (2.7) are identical with those connecting the coefficients in a "corresponding" continued fraction of Stieltjes and the "associated" continued fraction obtained by contraction (e.g., see $[5, \S 2])$. The $Q_{n}(x)$ are the "kernel polynomials" associated with the $P_{n}(x)$ and (2.6) and (2.7) have been given in this connection by Stiefel $[7, \S 5]$ (without reference to the $R_{n}(x)$, however). Although this usage of the term, "kernel polynomials," is perhaps somewhat more restrictive than what is customary in the theory of orthogonal polynomials (see $[9, \S 3.1]$ ), we will use it in this paper to mean the polynomials which are orthogonal with respect to a distribution, $x d \psi(x)$, where $\psi$ is a distribution function whose spectrum is an infinite subset of $[0, \infty)$.

From the above derivation, it is clear that a necessary and sufficient condition for the polynomials defined by (1.1) to be orthogonal on a subset of $(0, \infty)$ is the existence of $\gamma_{n}, \gamma_{1}=0, \gamma_{n}>0(n \geqq 2)$, satisfying (2.6). Similarly, (2.7) is a necessary and sufficient condition for the polynomials defined by (2.1) to be kernel polynomials.

Wall has studied sequences $\left\{a_{n}\right\}, 0 \leqq a_{n} \leqq 1(n \geqq 1)$, for which there exist numbers $g_{n}, 0 \leqq g_{n} \leqq 1$, such that $a_{n}=\left(1-g_{n-1}\right) g_{n}(n=1,2, \cdots)$. Wall calls such sequences, "chain sequences," and the $g_{k}$ the "parameters" of the sequence. A chain sequence does not, in general, uniquely determine its parameter sequence $\left(^{1}\right)\left\{g_{k}\right\}$. Indeed, Wall proves that every chain sequence has "minimal" and "maximal" parameter sequences, $\left\{m_{n}\right\}$ and $\left\{M_{n}\right\}$, such that if $\left\{h_{n}\right\}$ is any parameter sequence for $\left\{a_{n}\right\}$, then $m_{n} \leqq h_{n} \leqq M_{n}(n=0,1, \cdots)$, $m_{0}=0$.

In the remainder of this paper, chain sequences $\left\{a_{n}\right\}$, in which $0<a_{n}<1$, will play a fundamental role, and it will facilitate the work if we note here some fundamental results due to Wall $[10, \S \S 19$ and 20$]$, modifying the results in a few instances to take advantage of the additional restriction, 0 $<a_{n}<1$.

(W1) If $M_{0}>0$, then $0<m_{n}<M_{n}(n \geqq 1)$ and for every $h_{0}, 0<h_{0}<M_{0}$, there exists a corresponding parameter sequence, $\left\{h_{n}\right\}$, such that $m_{n}<h_{n}$ $<M_{n}$.

(W2) A necessary and sufficient condition for a parameter sequence $\left\{g_{n}\right\}$ to be a maximal parameter sequence is the divergence of the series

(1) Since it will always be clear when we mean a parameter sequence, no confusion should arise from writing $\left\{a_{n}\right\}$ rather than $\left\{a_{n}\right\}_{1}^{\infty}$ for a chain sequence and $\left\{g_{n}\right\}$ rather than $\left\{g_{n}\right\}_{0}^{\infty}$ for a parameter sequence. 


$$
\sum_{n=1}^{\infty} \frac{g_{1} g_{2} \cdots g_{n}}{\left(1-g_{1}\right)\left(1-g_{2}\right) \cdots\left(1-g_{n}\right)} .
$$

(W3) If $\left\{b_{n}\right\}$ is a chain sequence with a parameter sequence $\left\{h_{n}\right\}$, and if $a_{n} \leqq b_{n}(n \geqq 1)$, then $m_{n} \leqq h_{n} \leqq M_{n}(n \geqq 0)$.

(W4) If $0<c_{n} \leqq a_{n}(n \geqq 1)$, then $\left\{c_{n}\right\}$ is a chain sequence.

(W5) If $a_{n}=a \leqq 1 / 4(n \geqq 1)$, then $M_{n}=\left[1+(1-4 a)^{1 / 2}\right] / 2$ and $\lim _{n \rightarrow \infty} m_{n}$ $=\left[1-(1-4 a)^{1 / 2}\right] / 2$. Also, $0<m_{n}<m_{n+1}(n \geqq 1)$.

If $a>1 / 4$, then $\{a\}$ is not a chain sequence.

Turning now to the relations (2.6), we note that if the $\gamma_{n}$ exist, then $0<\gamma_{2 n+1}<c_{n+1}$ so there exist numbers $g_{n}, g_{0}=0,0<g_{n}<1(n>0)$, such that $\gamma_{2 n-1}=g_{n-1} c_{n}(n>0)$. Thus $\gamma_{2 n}=\left(1-g_{n-1}\right) c_{n}$ and $\lambda_{n+1} /\left(c_{n} c_{n+1}\right)=\left(1-g_{n-1}\right) g_{n}$ $(n \geqq 1)$. Thus $\left\{\lambda_{n+1} /\left(c_{n} c_{n+1}\right)\right\}$ is a chain sequence with minimal parameters, $g_{n}$.

Similarly, if $(2.7)$ holds, then there exist $h_{n}, 0<h_{n}<1(n \geqq 0)$, such that $\gamma_{2 n}=h_{n-1} d_{n}, \gamma_{2 n+1}=\left(1-h_{n-1}\right) d_{n}$ and hence $\left\{\nu_{n+1} /\left(d_{n} d_{n+1}\right)\right\}$ is a chain sequence with parameters $h_{n}$ which are not minimal since $h_{0} \neq 0$.

Conversely, given (1.1) with $c_{n}>0$ and such that $\left\{\lambda_{n+1} /\left(c_{n} c_{n+1}\right)\right\}$ is a chain sequence, then $\lambda_{n+1} /\left(c_{n} c_{n+1}\right)=\left(1-k_{n-1}\right) k_{n}(n \geqq 1)$ where, since $\lambda_{n+1}>0$, $0 \leqq k_{0}<1$ and $0<k_{n}<1 \quad(n>0)$. Then defining $\Gamma_{2 n-1}=k_{n-1} c_{n}$ and $\Gamma_{2 n}$ $=\left(1-k_{n-1}\right) c_{n}$, we have

$$
c_{n}=\Gamma_{2 n-1}+\Gamma_{2 n}, \quad \lambda_{n+1}=\Gamma_{2 n} \cdot \Gamma_{2 n+1} \quad(n=1,2, \cdots) .
$$

Thus if $k_{0}=0$, then we have (2.6) with $\gamma_{n}=\Gamma_{n}$, while if $k_{0}>0$, we have equations of the form (2.7) with $\gamma_{n+1}=\Gamma_{n}$. In the latter case, we can use equations of the form (2.6) to construct a set of orthogonal polynomials whose kernel polynomials are the $P_{n}(x)$ satisfying (1.1). Thus we have

TheOREM 1. Let $\left\{P_{n}(x)\right\}$ be defined by (1.1). A necessary and sufficient condition for the $P_{n}(x)$ to be orthogonal polynomials whose true interval of orthogonality is a subset of $(0, \infty)$ is that $c_{n}>0$ and $\left\{\lambda_{n+1} /\left(c_{n} c_{n+1}\right)\right\}$ is a chain sequence. In order that they be kernel polynomials, it is necessary and sufficient that $c_{n}>0$ and $\left\{\lambda_{n+1} /\left(c_{n} c_{n+1}\right)\right\}$ is a chain sequence which does not determine its parameters uniquely.

3. Further study of the kernel polynomials. The preceding suggests further study of the case where the chain sequence does not determine its parameters uniquely.

We first note that a recurrence formula of the form (1.1) determines a sequence of moments, $\mu_{n}$, up to the values of the ratios, $\mu_{n} / \mu_{0}$, and with an arbitrary choice of $\mu_{0}>0$, the corresponding Hamburger moment problem has a solution, $\psi$ (see Favard's proof [3]). The $P_{n}(x)$ are then orthogonal with respect to $d \psi(x)$ (and we will then speak of $\psi$ as a distribution function associated with $\left.\left\{P_{n}(x)\right\}\right)$. It will be convenient to assume henceforth that all distribution functions are normalized; that is, for any distribution function $\theta$ whose spectrum is a subset of $[a, b]$, 


$$
\theta(x)= \begin{cases}0 & x \leqq a, \\ {[\theta(x+0)+\theta(x-0)] / 2} & a<x<b, \\ \theta(b) & b \leqq x\end{cases}
$$

(with appropriate interpretations if $a$ or $b$ is infinite).

Since the moment problems are essentially equivalent, we will speak of "the" moment problem associated with (1.1). In case the moment problem is determined, $\psi$ is uniquely determined up to an arbitrary positive factor and we will then speak of "the" distribution function associated with $\left\{P_{n}(x)\right\}$.

Now consider $\left\{Q_{n}(x)\right\}$ defined by (2.1) and assume that $d_{n}>0$ and $\left\{\nu_{n+1} /\left(d_{n} d_{n+1}\right)\right\}$ is a chain sequence which does not determine its parameters uniquely. Let $M=\left\{M_{n}\right\}$ denote its maximal parameter sequence, and for each $h_{0}, 0<h_{0} \leqq M_{0}$, let $h=\left\{h_{n}\right\}$ denote the corresponding parameter sequence. Now define

$$
\begin{aligned}
\gamma_{1}^{h} & =0, \quad \gamma_{2 n}^{h}=h_{n-1} d_{n}, \quad \gamma_{2 n+1}^{h}=\left(1-h_{n-1}\right) d_{n} & & (n=1,2, \cdots), \\
c_{n}^{h} & =\gamma_{2 n-1}^{h}+\gamma_{2 n}^{h}, \quad \lambda_{n+1}^{h}=\gamma_{2 n}^{h} \gamma_{2 n+1}^{h} & & (n=1,2, \cdots) .
\end{aligned}
$$

Let $P_{n}^{\lambda}(x)$ denote the polynomials satisfying (1.1) with $c_{n}=c_{n}^{n}, \lambda_{n+1}=\lambda_{n+1}^{h}$. Then since the $d_{n}$ and $\nu_{n}$ clearly satisfy (2.7) with $\gamma_{n}=\gamma_{n}^{n}$, it follows that $\left\{Q_{n}(x)\right\}$ is the unique kernel set corresponding to $\left\{P_{n}^{h}(x)\right\}$ for every $h$. With the present notation, we now prove

TheOREM 2. The polynomials $P_{n}^{M}(x)$ are orthogonal on an interval $(a, b)$ $C(0, \infty)$ with respect to a distribution, $d \psi^{M}(x)$, where $\psi^{M}$ is the solution of a determined Hamburger moment problem and is continuous at the origin.

For $h \neq M$, the $P_{n}^{h}(x)$ are orthogonal on $(0, b)$ and a corresponding (normalized) distribution function, $\psi^{h}$, is furnished by

$$
\psi^{h}(x)=\psi^{M}(x)+\psi^{M}(b)\left(M_{0}-h_{0}\right) / h_{0}, \quad x>0 .
$$

The moment problem corresponding to $\psi^{h}$ is a determined Stieltjes moment problem if and only if $x d \psi^{M}(x)$ defines a solution of a determined Stieltjes moment problem.

Proof. With an obvious definition of $R_{n}^{h}(x)$, we have by (2.2) and (2.3),

$$
\begin{aligned}
& P_{n}^{h}(0)=R_{2 n}^{h}(0)=(-1)^{n} \gamma_{2}^{h} \gamma_{4}^{h} \cdots \gamma_{2 n}^{h}, \\
&\left|p_{n}^{h}(0)\right|^{2} \equiv\left[\lambda_{2}^{h} \lambda_{3}^{h} \cdots \lambda_{n+1}^{h}\right]^{-1}\left|P_{n}^{h}(0)\right|^{2}=\frac{\gamma_{2}^{h} \gamma_{4}^{h} \cdots \gamma_{2 n}^{h}}{\gamma_{3}^{h} \gamma_{5}^{h} \cdots \gamma_{2}^{h}{ }^{n+1}} \\
&=\frac{h_{0} h_{1} \cdots h_{n-1}}{\left(1-h_{0}\right) \cdots\left(1-h_{n-1}\right)}, \\
& {\left[\rho^{h}(0)\right]^{-1} \equiv \sum_{n=0}^{\infty}\left|p_{n}^{h}(0)\right|^{2}=1+\sum_{n=0}^{\infty} \frac{h_{0} h_{1} \cdots h_{n}}{\left(1-h_{0}\right) \cdots\left(1-h_{n}\right)} . }
\end{aligned}
$$


Now if $\rho^{h}(0)=0$, then $\left\{P_{n}^{h}(x)\right\}$ corresponds to a determined Hamburger moment problem and, moreover, the solution to the moment problem is continuous at 0 [6, Theorem 2.9, Corollary 2.6]. Hence by (W2), the first assertion of the theorem follows.

Now let $\psi^{h}$ be a distribution function associated with $\left\{P_{n}^{h}(x)\right\}$ with a spectrum which is a subset of $[0, \infty)$. Then we can assume without loss of generality that

$$
\mu_{1}^{h}=\mu_{1}^{M} \quad \text { where } \quad \mu_{n}^{h}=\int_{0}^{\infty} x^{n} d \psi^{h}(x) .
$$

Since $\left\{Q_{n}(x)\right\}$ is the unique kernel set associated with $\left\{P_{n}^{h}(x)\right\}$, then $x d \psi^{h}(x)$ furnishes a distribution for $\left\{Q_{n}(x)\right\}$. Choosing $\mu_{0}^{(1)}=\mu_{1}^{M}$, we uniquely determine the moments $\mu_{n}^{(1)}$ associated with $\left\{Q_{n}(x)\right\}$ and we have

$$
\mu_{n}^{(1)}=\int_{0}^{\infty} x^{n+1} d \psi^{h}(x)=\mu_{n+1}^{h} \quad(n=0,1,2, \cdots) .
$$

It follows that if $\left\{\mu_{n}^{(1)}\right\}$ defines a determined Stieltjes moment problem, then

$$
\int_{0}^{x} t d \psi^{h}(t)=\int_{0}^{x} t d \psi^{M}(t) \quad(x \geqq 0),
$$

whence for $x \geqq 0$,

$$
x \omega^{h}(x)=\int_{0}^{x} \omega^{h}(t) d t, \quad \omega^{h}(x)=\psi^{h}(x)-\psi^{M}(x) .
$$

Since $\omega^{h}$ is of bounded variation, it is continuous, hence absolutely continuous on every interval $[\epsilon, \delta], 0<\epsilon<\delta$, and therefore $D_{x} \omega^{h}(x)$ exists and vanishes for $x>0$. Thus

$$
\psi^{h}(x)=\psi^{M}(x)+J^{h}
$$

where $J^{h}=\psi^{h}(\infty)-\psi^{M}(\infty)=\mu_{0}^{h}-\mu_{0}^{M}$. But

$$
0=\int_{0}^{\infty} P_{1}^{h}(x) d \psi^{h}(x)=\mu_{1}^{h}-c_{1}^{h} \mu_{0}^{h}
$$

and $\mu_{1}^{h}=\mu_{1}^{M}$, so that $\mu_{0}^{h}=\mu_{1}^{M} / c_{1}^{h}=\mu_{0}^{M} c_{1}^{M} / c_{1}^{h}$. Thus

$$
J^{h}=\stackrel{M}{\mu_{0}^{M}}\left(c_{1}^{M} / c_{1}^{h}-1\right)=\stackrel{M}{\mu_{0}}\left(M_{0} / h_{0}-1\right) .
$$

Conversely, if $\left\{\mu_{n}^{(1)}\right\}$ defines an indeterminate Stieltjes moment problem, the preceding shows that (3.3) still furnishes a distribution function associated with $\left\{P_{n}(x)\right\}$. However, the corresponding moment problem for $h \neq M$ will also be an indeterminate Stieltjes moment problem as we now show.

According to a classical criterion of Hamburger, a necessary and sufficient 
condition that the Stieltjes moment problem corresponding to a sequence of moments $\left\{\mu_{n}\right\}$ be determined is that at least one of the definite forms

$$
\sum_{i, j=0}^{\infty} \mu_{i+j} x_{i} x_{j} \text { and } \sum_{i, j=0}^{\infty} \mu_{i+j+1} x_{i} x_{j}
$$

be "improperly definite" (see [6, p. 75]). Applying this criterion to $\mu_{n} \equiv \mu_{n}^{(1)}$ $=\mu_{n+1}^{h}$, it follows that $\sum \mu_{i+j+1}^{h} x_{i} x_{j}$ in particular must be "properly definite." But $\mu_{0}^{h}=\mu_{0}^{M}+J^{h}$ and $\mu_{i}^{h}=\mu_{i}^{M}(i>0)$ so that

$$
\sum_{i, j=0}^{\infty} \mu_{i+j}^{h} x_{i} x_{j}=J^{h} x_{0}^{2}+\sum_{i, j=0}^{\infty} \mu_{i+j}^{M} x_{i} x_{j} .
$$

It follows that $\sum \mu_{i+j}^{h} x_{i} x_{j}$ is also properly definite and $\left\{\mu_{n}^{h}\right\}(h \neq M)$ defines an indeterminate Stieltjes moment problem.

A simple example of the systems of polynomials discussed in Theorem 2 is provided by $Q_{n}(x)=4^{-n} T_{n}(2 x-1)$, where $T_{n}(x)$ is the Tchebichef polynomial of the first $\operatorname{kind}\left({ }^{2}\right)$. We have $d_{n}=1 / 2, \nu_{n+1}=1 / 16$, so by (W5), $M_{n}=1 / 2(n \geqq 0)$. It can then be readily verified that corresponding to $0<h_{0} \leqq 1 / 2$,

$$
h_{n}=2^{-1}\left[n\left(1-2 h_{0}\right)+1\right]^{-1}\left[n\left(1-2 h_{0}\right)+2 h_{0}\right] \quad(n=1,2, \cdots),
$$

from which the $\gamma_{n}^{n}, c_{n}^{h}$ and $\lambda_{n}^{h}$ can easily be calculated. We omit the results but note that for $h_{0}=M_{0}=1 / 2$, we have

$$
P_{n}^{M}(x)=(n !)^{2}[(2 n) !]^{-1} P_{n}^{(-r, r)}(2 x-1) \quad(r=1 / 2),
$$

where $P_{n}^{(\alpha, \beta)}(x)$ is the Jacobi polynomial.

We next observe a simple separation theorem for the zeros of the polynomials of Theorem 2 .

THEOREM 3. Let $h=\left\{h_{n}\right\}$ and $k=\left\{k_{n}\right\} \quad\left(0<h_{0}<k_{0}\right)$ be two parameter sequences for $\left\{\nu_{n+1} /\left(d_{n} d_{n+1}\right)\right\}$. Let $x_{n \nu}^{h}$ and $x_{n \nu}^{k}(\nu=1, \cdots, n)$ denote the zeros of $P_{n}^{h}(x)$ and $P_{n}^{k}(x)$, respectively, ordered according to ascending magnitude. Let $y_{n \nu}(\nu=1, \cdots, n)$ denote the similarly ordered zeros of $Q_{n}(x)$. Then we have

$$
0<x_{n \nu}^{h}<x_{n \nu}^{k}<y_{n \nu}<x_{n+1, \nu+1}^{h} \quad(\nu=1, \cdots, n) .
$$

Proof. From (2.4), it follows that $Q_{n}\left(x_{n \nu}^{i}\right)$ alternates in sign with $P_{n+1}^{i}\left(x_{n \nu}^{i}\right)$ and that $Q_{n}\left(x_{n+1, \nu}^{i}\right)$ alternates in sign with $P_{n}^{i}\left(x_{n+1, \nu}^{i}\right)(i=h, k)$. Thus by the classical separation theorem for the zeros of consecutive orthogonal polynomials, it follows that the zeros of $Q_{n}(x)$ separate those of both $P_{n}^{i}(x)$ and $P_{n+1}^{i}(x)$. Also, $Q_{n}\left(x_{n n}^{i}\right)<0$ and $Q_{n}\left(x_{n+1, n+1}^{i}\right)>0$ since $x_{n n}^{i}<x_{n+1, n+1}^{i}$. Thus

$$
x_{n \nu}^{i}<y_{n \nu}<x_{n+1, \nu+1}^{i} \quad(\nu=1, \cdots, n ; i=h, k) .
$$

(2) Unless otherwise noted, the notation of [9] will be used for particular classes of orthogonal polynomials. 
From (2.5) we have

$$
P_{n}^{h}(x)-P_{n}^{k}(x)=\left(\gamma_{2 n+1}^{h}-\gamma_{2 n+1}^{k}\right) Q_{n-1}(x) .
$$

By (3.1) and (W1), $\gamma_{2 n+1}^{n}=d_{n}\left(1-h_{n-1}\right)>d_{n}\left(1-k_{n-1}\right)=\gamma_{2 n+1}^{k}$. Therefore, $P_{n}^{h}\left(x_{n \nu}^{k}\right)$ agrees in sign with $Q_{n-1}\left(x_{n v}^{k}\right)$ which in turn agrees in sign with $P_{n-1}^{k}\left(x_{n \nu}^{k}\right)$. Hence $x_{n \nu}^{h}<x_{n \nu}^{k}$ which combined with (3.5) yields (3.4).

REMARK. The conclusions concerning the mutual separation of the zeros could also be drawn by making the observations that $x Q_{n}(x)$ is a quasiorthogonal polynomial of order $n+1$ with respect to the distribution, $d \psi^{i}(x)$, and that $P_{n}^{i}(x)$ is a quasi-orthogonal polynomial of order $n$ with respect to the distribution, $x d \psi^{M}(x)$ (see [6, p. 36]).

4. Some results on chain sequences. We interrupt our study of (1.1) to establish a few results on chain sequences which will be needed later. Throughout this section, $\left\{a_{n}\right\}$ denotes a chain sequence with $0<a_{n}<1$, and $\left\{m_{n}\right\}$ and $\left\{M_{n}\right\}$ denote its minimal and maximal parameter sequences. We also use the notation, $c_{n}^{(\boldsymbol{k})}=c_{n+k}$.

LEMMA 1. For every fixed integer $k \geqq 0$ :

(a) $\left\{a_{n}^{(\mathbf{k})}\right\}$ is a chain sequence and it has the parameter sequence $\left\{g_{n}^{(\mathbf{k})}\right\}$, where $\left\{g_{n}\right\}$ is any parameter sequence for $\left\{a_{n}\right\}$;

(b) the maximal parameter sequence for $\left\{a_{n}^{(\mathbf{k})}\right\}$ is $\left\{M_{n}^{(\mathbf{k})}\right\}$;

(c) the minimal parameters, $m_{k n}$, of $\left\{a_{n}^{(k)}\right\}$ satisfy $m_{k n}<m_{n}^{(k)}$.

Proof. (a) is obvious; (b) follows from (a) and (W2); (c) follows from (a) and (W1) which shows that $m_{0}^{(k)}>0$.

Lемма 2. Assume $\left\{a_{n}\right\}$ does not determine its parameters uniquely, and let $\left\{h_{n}\right\}$ be any parameter sequence such that $m_{n} \leqq h_{n}<M_{n}$. Then $\lim _{n \rightarrow \infty} m_{n} / h_{n}=1$.

Proof. $a_{n}=\left(1-m_{n-1}\right) m_{n}=\left(1-h_{n-1}\right) h_{n}$, hence

$$
\begin{aligned}
\delta_{n} & \equiv h_{n}-m_{n}=h_{n} \delta_{n-1}+m_{n-1} \delta_{n}, \\
0 \leqq \delta_{n} & =h_{n} \delta_{n-1} /\left(1-m_{n-1}\right) \\
& =\prod_{k=1}^{n} \frac{h_{k}}{1-m_{k-1}} \delta_{0}=h_{n} \prod_{k=0}^{n-1} \frac{h_{k}}{1-m_{k}} \quad(n \geqq 1) .
\end{aligned}
$$

Thus

$$
0 \leqq 1-\frac{m_{n}}{h_{n}}=\prod_{k=0}^{n-1} \frac{h_{k}}{1-m_{k}}<\prod_{k=0}^{n-1} \frac{h_{k}}{1-h_{k}}
$$

According to (W2), the last expression tends to zero as $n \rightarrow \infty$ which proves the lemma.

The conclusion of Lemma 2 need not hold if $h=M$ as (W5) shows. Indeed, we have 
LemMA 3. $\lim _{n \rightarrow \infty} m_{n} / M_{n}=L<1$ if and only if $\lim _{n \rightarrow \infty} M_{n}=M$ and $\lim _{n \rightarrow \infty} m_{n}=m$ where $1 / 2<M \leqq 1$ and $m=1-M$ (hence also $\lim _{n \rightarrow \infty} a_{n}$ $=(1-M) M<1 / 4)$.

Proof. As in Lemma 2,

$$
0<1-\frac{m_{n}}{M_{n}}=\prod_{k=0}^{n-1} \frac{M_{k}}{1-m_{k}},
$$

so that $\lim _{n \rightarrow \infty} m_{n} / M_{n}=L<1$ implies $\lim _{k \rightarrow \infty} M_{k} /\left(1-m_{k}\right)=1$ which in turn implies $\lim _{n \rightarrow \infty}\left(M_{n}+m_{n}\right)=1$. This together with $\lim _{n \rightarrow \infty} m_{n} / M_{n}=L$ implies $\lim _{n \rightarrow \infty} M_{n}=(1+L)^{-1}, \lim _{n \rightarrow \infty} m_{n}=L(1+L)^{-1}$.

The converse is obvious.

LEMMA 4. $\lim _{n \rightarrow \infty} a_{n}=a$ if and only if $0 \leqq a \leqq 1 / 4$ and $\lim _{n \rightarrow \infty} M_{n}$ $=\left[1+(1-4 a)^{-1 / 2}\right] / 2$. Moreover, if $M_{0}>0$, then $\lim _{n \rightarrow \infty} m_{n}=\left[1-(1-4 a)^{-1 / 2}\right] / 2$.

Proof. Assume first that $0<a<1 / 4$ and select $\epsilon>0$ such that $0<a-\epsilon<$ $a+\epsilon<1 / 4$. Then for $k=k(\epsilon)$ sufficiently large,

$$
a-\epsilon<a_{n}^{(k)}<a+\epsilon \quad(n \geqq 0) .
$$

Let $m_{n}^{\prime}$ and $M_{n}^{\prime}, m_{n}^{\prime \prime}$ and $M_{n}^{\prime \prime}$, denote the $n$th minimal and maximal parameters, respectively, of the chain sequences, $\{a-\epsilon\}$ and $\{a+\epsilon\}$. Then by (W3), we have

$$
m_{n}^{\prime} \leqq m_{k n} \leqq m_{n}^{\prime \prime}<M_{n}^{\prime \prime} \leqq M_{n}^{(k)} \leqq M_{n}^{\prime} \quad(n \geqq 0),
$$

where $m_{k n}$ has the same meaning as in Lemma 1. Thus by (W5)

$$
\begin{aligned}
\frac{1}{2}\left\{1+[1-4(a-\epsilon)]^{1 / 2}\right\} \leqq \liminf _{n \rightarrow \infty} & M_{n}^{(k)} \leqq \limsup _{n \rightarrow \infty} M_{n}^{(k)} \\
& \leqq \frac{1}{2}\left\{1+[1-4(a+\epsilon)]^{1 / 2}\right\}
\end{aligned}
$$

Since $M_{n}^{(k)}=M_{n+k}$, it follows that $\lim _{n \rightarrow \infty} M_{n}=\left[1+(1-4 a)^{1 / 2}\right] / 2$. Similarly, it follows that $\lim _{n \rightarrow \infty} m_{k n}=\left[1-(1-4 a)^{1 / 2}\right] / 2$. Therefore, if $\left\{a_{n}\right\}$ does not determine its parameters uniquely $\left(M_{0}>0\right)$, then $\left\{m_{n}^{(k)}\right\}$ is a nonmaximal parameter sequence for $\left\{a_{n}^{(k)}\right\}$ and by Lemma 2 , the desired conclusion for $\left\{m_{n}\right\}$ follows.

The case $a=0$ is handled in the same way except that $a-\epsilon$ is replaced by 0 . The case $a=1 / 4$ is similarly treated using only the inequality, $1 / 4-\epsilon$ $<a_{k}^{(n)}$, which leads to

$$
m_{n}^{\prime} \leqq m_{n}^{(k)} \leqq M_{n}^{(k)} \leqq\left[1+(4 \epsilon)^{1 / 2}\right] / 2,
$$

from which the desired conclusion follows. 
Again, the converse is obvious.

Comparing Lemmas 3 and 4 , we see that if $\left\{a_{n}\right\}$ does not determine its parameters uniquely, then

$$
\lim _{n \rightarrow \infty} a_{n}=a \leqq 1 / 4 \Rightarrow \lim _{n \rightarrow \infty} m_{n} / M_{n}=L \leqq 1 \quad\left(a=L(1+L)^{-2}\right),
$$

while the reverse implication holds if $L<1(a<1 / 4)$. The following example shows that the reverse implication need not hold if $L=1$.

Let

$$
a_{1}=1 / 4, \quad a_{2 n}=(n+1)^{-2}, \quad a_{2 n+1}=n(n+2)^{-1} \quad(n \geqq 1) .
$$

Then

$$
\begin{aligned}
& m_{0}=0, \quad m_{2 n-1}=n^{2}\left(n^{2}+n+2\right)^{-1}, \quad m_{2 n}=\left(n^{2}+n+2\right)(n+2)^{-1}(n+1)^{-2}, \\
& M_{0}=1 / 2, \quad M_{2 n-1}=n(n+1)^{-1}, \quad M_{2 n}=(n+1)^{-1} \quad(n \geqq 1) .
\end{aligned}
$$

Thus $\lim _{n \rightarrow \infty} m_{n} / M_{n}=1$ while $\lim _{n \rightarrow \infty} a_{n}$ does not exist.

5. On the true interval of orthogonality. We now return to the polynomials defined by (1.1) and consider their true interval of orthogonality, $(a, b)$.

Suppose first that $a$ is finite. Then the polynomials defined by $P_{n, 1}(x)$ $=P_{n}(x+a)$ are orthogonal on $(0, b-a)$ and satisfy

$$
P_{n, 1}(x)=\left(x-c_{n}^{\prime}\right) P_{n-1,1}(x)-\lambda_{n} P_{n-2,1}(x), \quad c_{n}^{\prime}=c_{n}-a,
$$

so that $c_{n}>a$ and $\left\{\lambda_{n+1} /\left(c_{n}^{\prime} c_{n+1}^{\prime}\right)\right\}$ is a chain sequence.

Similarly, if $b$ is finite, we consider the polynomials, $P_{n, 2}(x)$ $=(-1)^{n} P_{n}(b-x)$, which are orthogonal on $(0, b-a)$, and conclude that $b>c_{n}$ and that $\left\{\lambda_{n+1} /\left(c_{n}^{\prime \prime} c_{n+1}^{\prime \prime}\right)\right\}$, where $c_{n}^{\prime \prime}=b-c_{n}$, is a chain sequence.

Thus we clearly have

Lemma 5. Let $(a, b)$ denote the true interval of orthogonality of $\left\{P_{n}(x)\right\}$. Then $a$ is the maximum value of $c$ for which $c_{n}>c$ and $\left\{\lambda_{n+1} /\left[\left(c_{n}-c\right)\left(c_{n+1}-c\right)\right]\right\}$ is a chain sequence, while $b$ is the minimum value of $d$ for which $d>c_{n}$ and $\left\{\lambda_{n+1} /\left[\left(d-c_{n}\right)\left(d-c_{n+1}\right)\right]\right\}$ is a chain sequence. If such $c(d)$ do not exist, then $a(b)$ is $-\infty(+\infty)$.

Thus, for example, $(a, b)=(-\infty, \infty)$ in case either

$$
\limsup _{n \rightarrow \infty} c_{n}=-\liminf _{n \rightarrow \infty} c_{n}=\infty \text {, }
$$

or

$$
\underset{n \rightarrow \infty}{\lim \sup } \lambda_{n}=\infty \quad \text { and } \underset{n \rightarrow \infty}{\lim \inf } \lambda_{n+1} /\left(c_{n} c_{n+1}\right)>1 / 4
$$

In the latter case, the assertion follows from (W4) and Lemma 4.

On the other hand, $(a, b)$ is bounded if and only if both $\left\{c_{n}\right\}$ and $\left\{\lambda_{n}\right\}$ are bounded (as is known).

We turn now to the case where $a$ is finite. Throughout the remainder of this paper, the following notation will be used. 
For any number sequence $\left\{a_{n}\right\}, a_{n}^{(k)}=a_{n+k}$.

$P_{n}^{(k)}(x)$ denotes the $n$th polynomial satisfying (1.1) with $c_{n}$ and $\lambda_{n}$ replaced by $c_{n}^{(k)}$ and $\lambda_{n}^{(k)}$, respectively; $P_{n}^{(0)}(x)=P_{n}(x)$. (Thus the $P_{n}^{(k)}(x)$ are the "numerator polynomials" [5] of the $P_{n}^{(k-1)}(x)$.)

$\left(a^{(k)}, b^{(k)}\right)$ denotes the true interval of orthogonality of $\left\{P_{n}^{(k)}(x)\right\} ;\left(a^{(0)}, b^{(0)}\right)$ $=(a, b)$.

$\alpha_{n}=\lambda_{n+1} /\left(c_{n} c_{n+1}\right) ; \alpha=\left\{\alpha_{n}\right\}$.

$\mathcal{C}$ denotes the class of all chain sequences, $\left\{a_{n}\right\}$, such that $0<a_{n}<1$. If $b \in \mathcal{C}$, then $m_{n}(b)$ and $M_{n}(b)$ denote the $n$th minimal and maximal parameters of $b$ and we write $\delta_{n}(b)=M_{n}(b)-m_{n}(b)$.

Lemma 6. Let $\alpha \in \mathfrak{e}, c_{n}>0(n \geqq 1)$. Then

$$
c_{*}\left\{1-\left[4 \alpha^{*} /\left(\delta_{*}^{2}+4 \alpha^{*}\right)\right]^{1 / 2}\right\} \equiv C \leqq a \leqq c_{*},
$$

where $c_{*}=\inf \left\{c_{n}\right\}, \alpha^{*}=\sup \left\{\alpha_{n}\right\}, \delta_{*}=\inf \left\{\delta_{n}(\alpha)\right\}$.

Proof. The inequality, $a \leqq c_{*}$, is obvious since by Lemma 5 we must have $c_{n}>a$ for all $n$. The first inequality is also trivial if $c_{*}=0$; hence assume $c_{*}>0$ and let

$$
\alpha_{n}(c)=\lambda_{n+1} /\left[\left(c_{n}-c\right)\left(c_{n+1}-c\right)\right], \quad 0 \leqq c<c_{*} .
$$

Then

$$
\alpha_{n}(c)-\alpha_{n}=c \alpha_{n}\left\{\frac{1}{c_{n}-c}+\frac{1}{c_{n+1}-c}+\frac{c}{\left(c_{n}-c\right)\left(c_{n+1}-c\right)}\right\} .
$$

A little calculation now shows that for $c \leqq C$,

$$
\alpha_{n}(c)-\alpha_{n} \leqq c \alpha^{*}\left(2 c_{*}-c\right)\left(c_{*}-c\right)^{-2} \leqq \delta_{*}^{2} / 4
$$

hence

$$
0 \leqq \alpha_{n}(c) \leqq \alpha_{n}+\delta_{*}^{2} / 4 \leqq \alpha_{n}+\delta_{n-1}(\alpha) \delta_{n}(\alpha) / 4 \equiv \beta_{n},
$$

where $\beta_{n}=\left(1-g_{n-1}\right) g_{n}$ and $g_{n}=\left(M_{n}+m_{n}\right) / 2$. Thus by (W4), $\left\{\alpha_{n}(c)\right\} \in \mathbb{e}$ for $c \leqq C$ and hence by Lemma $5, a \geqq C$.

REMARK. The example, $c_{n}=k>0, \lambda_{n+1}=\lambda k^{2}, 0<\lambda<1 / 4$, shows that the equality, $a=C$, is possible since we then have

$$
\alpha^{*}=\alpha_{n}=\lambda<1 / 4, \quad \delta_{*}=(1-4 \lambda)^{1 / 2}\left(\text { by (W5)), } \quad C=k\left(1-2 \lambda^{1 / 2}\right),\right.
$$

while

$$
P_{n}(x)=2^{-n} U_{n}(t), \quad t=(x-k) /\left(2 k \lambda^{1 / 2}\right),
$$

where $U_{n}(x)$ is the Tchebichef polynomial of the second kind.

Theorem 4. Let $\alpha \in \mathfrak{C}, c_{n}>0(n \geqq 1)$.

(a) If $\inf \left\{c_{n}\right\}=0$, then $a=0$; 
(b) If $\inf \left\{c_{n}\right\}>0$ and $\sup \left\{c_{n}\right\}<\infty$, then a necessary and sufficient condition for $a=0$ is $\inf \left\{\delta_{n}(\alpha)\right\}=0$.

If $\sup \left\{c_{n}\right\}=\infty, \delta_{0}>0$, this condition is only necessary.

Proof. (a) follows trivially from Lemma 6. Hence suppose $\inf \left\{c_{n}\right\}>0$. Then by Lemma 6 again, $a=0$ implies $\inf \left\{\delta_{n}(\alpha)\right\}=0$.

Conversely, if $\inf \left\{\delta_{n}(\alpha)\right\}=0$ and $\sup \left\{c_{n}\right\}<\infty$, then either $\delta_{0}=0$, in which case $a=0$ by Theorem 1 , or $\delta_{n}(\alpha)>0$ for all $n$. In the latter case, consider a chain sequence, $\left\{\beta_{n}\right\}$, such that $\alpha_{n} \leqq \beta_{n}<1$. Let $\beta_{n}=\left(1-h_{n-1}\right) h_{n}$, $\beta_{n} / \alpha_{n}=1+\epsilon_{n}\left(\epsilon_{n}>0\right)$. By (W3), $m_{n} \equiv m_{n}(\alpha) \leqq h_{n} \leqq M_{n}(\alpha) \equiv M_{n}$, and we must have

$$
\left.1+\epsilon_{n}=\left(1-h_{n-1}\right) h_{n} /\left[1-m_{n-1}\right) m_{n}\right] \leqq h_{n} / m_{n} ;
$$

hence

$$
m_{n} \leqq \frac{h_{n}}{1+\epsilon_{n}}
$$

Also,

$$
1+\epsilon_{n}=\left(1-h_{n-1}\right) h_{n} /\left[\left(1-M_{n-1}\right) M_{n}\right] \leqq\left(1-h_{n-1}\right) /\left(1-M_{n-1}\right),
$$

hence

$$
M_{n-1} \geqq \frac{\epsilon_{n}+h_{n-1}}{1+\epsilon_{n}}
$$

Therefore

$$
\delta_{n}(\alpha) \geqq \frac{\epsilon_{n+1}+h_{n}}{1+\epsilon_{n+1}}-\frac{h_{n}}{1+\epsilon_{n}} \geqq \frac{e_{n}}{1+e_{n}},
$$

where $e_{n}=\min \left(\epsilon_{n}, \epsilon_{n+1}\right)$. It follows that $\operatorname{if} \inf \left\{\delta_{n}(\alpha)\right\}=0$, then

$$
\inf \left\{\frac{e_{n}}{1+e_{n}}\right\}=\inf \left\{\frac{\epsilon_{n}}{1+\epsilon_{n}}\right\}=\inf \left\{1-\frac{\alpha_{n}}{\beta_{n}}\right\}=0 .
$$

But by (5.1), $\inf \left\{\alpha_{n}(c) / \alpha_{n}\right\}>1$ for $c>0$. It follows that $\left\{\alpha_{n}(c)\right\} \notin \mathcal{C}$ for any $c>0$, hence by Lemma $5, a=0$.

REMARK. That the condition in (b) is not sufficient for $a=0$ when $\sup \left\{c_{n}\right\}$ $=\infty$ can be seen by considering the polynomials, $P_{n}(x)=(-1)^{n} n ! L_{n}(x-a)$, where $a>0$ and $L_{n}(x)$ is the Laguerre polynomial. In this case, we have $\alpha \in \mathcal{C}, \lim _{n \rightarrow \infty} \alpha_{n}=1 / 4$, so that $\inf \left\{\delta_{n}(\alpha)\right\}=0$ although $a>0$.

LEMMA 7. $a^{(k-1)} \leqq a^{(k)}(k \geqq 1)$. If the Hamburger moment problem associated with $\left\{P_{n}(x)\right\}$ is determined and if $\psi$ is the associated distribution function, the spectrum of $\psi$ contains at most $k$ points less than $a^{(k)}$; hence the spectrum of $\psi$ has no limit points less than $\lim _{k \rightarrow \infty} a^{(k)}$. 
Proof. The inequality, $a^{(k-1)} \leqq a^{(k)}$, is known [5] and follows from the well known facts that the zeros of orthogonal polynomials are interior to their interval of orthogonality and that the zeros of $P_{n-1}^{(\mathbf{k})}(x)$ separate those of $P_{n}^{(k-1)}(x)$. It also follows that $P_{n}^{(k-1)}(x)$ has at most one zero smaller than $a^{(k)}$. Thus $P_{n}(x)$ has at most $k$ zeros less than $a^{(k)}$. Since for a determined moment problem every point of the spectrum of $\psi$ is a limit point of the zeros of the $P_{n}(x)[6$, Lemma 4.3], it follows that the spectrum of $\psi$ contains at most $k$ points smaller than $a^{(k)}$.

THEOREM 5. Let $\alpha \in \mathfrak{C}, c_{n}>0(n \geqq 1)$.

If $\sup \left\{c_{n}\right\}<\infty$, then a necessary and sufficient condition for the distribution function $\psi$ associated with $\left\{P_{n}(x)\right\}$ to have a jump at 0 and be constant on an interval $\left(0, a^{\prime}\right), a^{\prime}>0$, is

$$
\inf \left\{c_{n}\right\}>0, \quad \delta_{0}=0, \quad \inf \left\{M_{n}^{(1)}-m_{1 n}\right\}>0,
$$

where, as in Lemma 1, $m_{1 n}=m_{n}\left(\alpha^{(1)}\right), \alpha^{(1)}=\left\{\alpha_{n}^{(1)}\right\}$.

If $\sup \left\{c_{n}\right\}=\infty$ and if the associated Hamburger moment problem is determined, (5.2) is sufficient but not necessary.

Proof. Let $\sup \left\{c_{n}\right\}<\infty$ so that $(a, b)$ is bounded and the associated moment problem is determined. If $\psi$ has the property stated in the theorem, then by Theorem 1, $\left\{\alpha_{n}\right\}$ determines its parameters uniquely; that is, $\delta_{0}=0$.

Since $\psi$ is constant on $\left(0, a^{\prime}\right), P_{n}(x)$ has at most one zero in $\left(0, a^{\prime}\right)[9$, Theorem 3.41.2]. Hence, as in the proof of Lemma 7, the spectrum of the distribution function, $\psi^{(1)}$, associated with $\left\{P_{n}^{(1)}(x)\right\}$ contains at most one point less than $a^{\prime}$. But $\psi$ and $\psi^{(1)}$ cannot have a common discontinuity [1, Theorem 3], hence it follows that $a^{(1)}>0$. Then by Theorem $4(\mathrm{a}), \inf \left\{c_{n}^{(1)}\right\}$ $>0$, and therefore by Theorem $4(\mathrm{~b}), \inf \left\{M_{n}^{(1)}-m_{1 n}\right\}=0$. Since by hypothesis, $c_{1}>0$, the necessity of $(5.2)$ is proved.

Conversely, suppose the conditions of (5.2) are satisfied. Then by Theorem $1, a=0$, and by Theorem $4, a^{(1)}>0$. Therefore, if we are dealing with a determined Hamburger moment problem, by Lemma $7, \psi$ must be constant on $\left(0, a^{(1)}\right)$ and have a jump at 0 .

REMARK. If we consider a set of polynomials such as the Laguerre polynomials with the interval of orthogonality translated to, say, $(1, \infty)$, then we can construct a corresponding set of co-recursive orthogonal polynomials whose true interval of orthogonality is $(0, \infty)$ [1]. The associated distribution function would then be constant on $(0,1)$ and have a jump at 0 and be the solution of a determined moment problem. However, for the Laguerre polynomials, and hence for the co-recursive polynomials, we have $\lim _{n \rightarrow \infty} \alpha_{n}=1 / 4$ so by Lemma $4, \lim _{n \rightarrow \infty}\left[M_{n}^{(1)}-m_{1 n}\right]=0$ which shows that the conditions of Theorem 5 are not necessary in the case of an infinite interval.

6. Some special cases. We consider some special hypotheses on $\left\{c_{n}\right\}$ and 
$\left\{\lambda_{n}\right\}$ for which the associated distribution functions have denumerable spectra. Throughout the remainder of the paper, we assume that (1.1) is associated with a determined Hamburger moment problem and denote by $\psi$ the corresponding distribution function which is uniquely determined up to an arbitrary positive factor.

Theorem 6. Let $\sigma$ and $\tau$ denote the least and greatest limit points of the spectrum of $\psi$. Then

$$
\sigma \leqq \liminf _{n \rightarrow \infty} c_{n} \leqq \limsup _{n \rightarrow \infty} c_{n} \leqq \tau
$$

Proof. Assume $\sigma$ and $\tau$ are finite. Then given $\epsilon>0, \psi$ has only finitely many points of increase on the complement of $[\sigma-\epsilon, \tau+\epsilon]$. Let $\xi_{1}<\xi_{2}<\ldots$ $<\xi_{p}$ denote the points of increase (if any) of $\psi$ which are less than $\sigma-\epsilon$. Then $\psi$ is constant on $\left(\xi_{i}, \xi_{i+1}\right)$ and $\left(\xi_{p}, \sigma-\epsilon\right)$, hence $P_{n}(x)$ has at most one zero on each of these intervals. Thus if $x_{n, j}$ are the zeros of $P_{n}(x)$ as in Theorem 3 , then since $\xi_{i}$ is a limit point of the zeros,

$$
\xi_{i}<x_{n, i} \text { and } \quad \lim _{n \rightarrow \infty} x_{n, i}=\xi_{i} \quad(i=1, \cdots, p) .
$$

Thus there exists $N_{1}=N_{1}(\epsilon)$ such that

$$
-\epsilon<\sum_{i=1}^{p}\left(x_{n, i}-x_{n-1, i}\right)<0 \quad\left(n \geqq N_{1}\right) .
$$

If the $\xi_{i}$ do not exist, we take $p=0$ and neglect (6.1).

Similarly, if $\psi$ has points of increase $\eta_{q-1}<\cdots<\eta_{1}<\eta_{0}$ greater than $\tau+\epsilon$, we conclude as before that

$$
x_{n, n-j}<\eta_{j} \quad \text { and } \quad \lim _{n \rightarrow \infty} x_{n, n-j}=\eta_{j} \quad(j=0, \cdots, q-1),
$$

and hence that there exists $N_{2}=N_{2}(\epsilon)$ such that

$$
0<\sum_{j=0}^{q-1}\left(x_{n, n-j}-x_{n-1, n-1-j}\right)<\epsilon \quad\left(n \geqq N_{2}\right),
$$

where we take $q=0$ and neglect (6.2) if the $\eta_{j}$ do not exist.

Finally, we have for $n \geqq \max \left(N_{1}, N_{2}\right)$

$$
\sigma-\epsilon<x_{n, p+1}<x_{n-1, p+1}<x_{n, p+2}<\cdots<x_{n-1, n-q-1}<x_{n, n-q}<\tau+\epsilon,
$$

hence

$$
\sigma-\epsilon<\sum_{k=p+1}^{n-q} x_{n, k}-\sum_{k=p+1}^{n-q-1} x_{n-1, k}<\tau+\epsilon .
$$

Combining (6.1), (6.2), and (6.3), we conclude that

$$
\sigma \leqq \liminf _{n \rightarrow \infty} \Sigma_{n} \leqq \limsup _{n \rightarrow \infty} \Sigma_{n} \leqq \tau,
$$


where $\Sigma_{n}=\sum_{i=1}^{n} x_{n, i}-\sum_{j=1}^{n-1} x_{n-1, j}$. But by (1.1), the coefficient of $x^{n-1}$ in $P_{n}(x)$ is $-\sum_{k=1}^{n} c_{k}$, hence $\Sigma_{n}=c_{n}$ so (6.4) yields the desired result.

If $\sigma$ or $\tau$ is infinite, the above proof requires only some notational modification.

TheORem 7. Let $\lim _{n \rightarrow \infty} \lambda_{n}=0$, let $\sigma$ and $\tau$ have the same meaning as in Theorem 6. Then

$$
\sigma=\liminf _{n \rightarrow \infty} c_{n} \quad \text { and } \quad \limsup _{n \rightarrow \infty} c_{n}=\tau .
$$

Proof. Suppose $\lim \inf _{n \rightarrow \infty} c_{n}=C_{*}>-\infty$. Then there exists $s \geqq-C_{*}$ such that $c_{n}^{\prime}=c_{n}+s>0$ and $\left\{\lambda_{n+1} /\left(c_{n}^{\prime} c_{n+1}^{\prime}\right)\right\} \equiv\left\{\alpha_{n}(-s)\right\} \in \mathbb{C}$. The corresponding polynomials, $P_{n}(x-s)$, are then orthogonal on $(a+s, b+s) \subset(0, \infty)$ with respect to the distribution, $d \psi_{1}(x)=d \psi(x-s)$. Now if $\lim _{\inf _{n \rightarrow \infty}}\left(c_{n}+s\right)=0$, then by Theorem $4, a+s=0$, hence $C_{*}=-s=a \leqq \sigma$. Otherwise, $C_{*}+s>0$ and in this case, $\left\{\alpha_{n}^{(k)}(-s)\right\}$ is a null sequence for every $k$. Then by Lemma 6 applied to $\left\{\alpha_{n}^{(\boldsymbol{k})}(-s)\right\}$,

$$
\inf _{n}\left\{\left(c_{n}^{(k)}+s\right)\right\} \theta_{k}(s) \leqq a^{(k)}+s \leqq \inf _{n}\left\{\left(c_{n}^{(k)}+s\right)\right\}
$$

where, since we have a null sequence, $\theta_{k}(s)$ is an expression which tends to 1 as $k \rightarrow \infty$. Thus $\lim _{k \rightarrow \infty} a^{(k)}=C_{*}$ and by Lemma $7, C_{*} \leqq \sigma$.

If $\lim \sup _{n \rightarrow \infty} c_{n}=C^{*}<\infty$, we consider the chain sequence $\left\{\lambda_{n+1} /\left[\left(t-c_{n}\right)\left(t-c_{n+1}\right)\right]\right\}$ corresponding to $\psi_{2}(x)=\psi_{2}(t-x)$, where $t$ is a sufficiently large number, and conclude that $\psi_{2}$ has at most denumerably many points of increase less than $t-C^{*}$ from which it follows that $t-\tau$ $\geqq t-C^{*}$. Thus we have

$$
C_{*} \leqq \sigma \leqq \tau \leqq C^{*}
$$

which combined with Theorem 6 establishes the theorem.

As an immediate corollary to Theorems 6 and 7, we have the following result which is equivalent to a theorem of Stieltjes on continued fractions $[3, \mathrm{pp} .560-566]$, the equivalence following from the well known relation between the continued fraction (1.2) and the distribution function for the corresponding orthogonal polynomials. (Dickinson, Pollak and Wannier [2] have recently given a constructive proof, without reference to continued fractions, of a special case of the sufficiency portion of this theorem.)

Theorem [STIELTJES]. Let $\left\{R_{n}(x)\right\}$ denote a set of orthogonal polynomials defined by (2.3). Then a necessary and sufficient condition for the associated distribution function $\phi$ to have a denumerable spectrum whose only limit point is 0 is $\lim _{n \rightarrow \infty} \gamma_{n}=0$.

Proof. The sufficiency follows immediately from Theorem 7 by taking $c_{n}=0, \lambda_{n+1}=\gamma_{n+1}(n \geqq 1)$. To prove the necessity, we consider the polynomials, $P_{n}(x)$, related to the $R_{n}(x)$ by (2.2). Since the spectrum of the distribution 
function associated with the $P_{n}(x)$ has 0 as its only limit point, we conclude from Theorem 6 that $\lim _{n \rightarrow \infty} c_{n}=0$ from which, by (2.6), the theorem follows.

According to Theorem 7 , if $\lim _{n \rightarrow \infty} c_{n}=c$ (and $\lim _{n \rightarrow \infty} \lambda_{n}=0$ ), then the spectrum of $\psi$ is a denumerable set whose only limit point is $c$. This holds in particular if $c=\infty$. However, in this case we have the following more general result.

TheOREM 8. Let $\lim _{n \rightarrow \infty} c_{n}=\infty$ and $\lim \sup _{n \rightarrow \infty} \lambda_{n+1} /\left(c_{n} c_{n+1}\right)<1 / 4$. Then the spectrum of $\psi$ is a denumerable set whose only limit point is $\infty$. (As throughout this section, we assume that the associated Hamburger moment problem is determined.)

Proof. For a sufficiently large $s, c_{n}+s>0, \alpha_{n}(-s) \leqq r<1 / 4(n \geqq 1)$, where $\alpha_{n}(-s)$ is defined as in Lemma 6. Thus by (W4) and (W5), $\left\{\alpha_{n}(-s)\right\} \in \mathfrak{C}$ and we have (6.5) again where by (W5) inf $\left\{\theta_{k}\right\} \geqq R>0$. Then $\lim _{k \rightarrow \infty} a^{(k)}=\infty$ and the theorem follows from Lemma 7.

It is interesting to compare the familiar Laguerre and Charlier polynomials with the orthogonal polynomials, studied by Gottlieb [4], which satisfy (1.1) with $c_{n+1}=\left(n e^{\lambda}+n+1\right)\left(e^{\lambda}-1\right)^{-1}, \lambda_{n+1}=n^{2} e^{\lambda}\left(e^{\lambda}-1\right)^{-2}(\lambda>0)$. In the Laguerre and Charlier cases, $\lim _{n \rightarrow \infty} \alpha_{n}=1 / 4$ and 0 , respectively, while in the Gottlieb case, the corresponding limit is $e^{\lambda}\left(e^{\lambda}+1\right)^{-2}$. Thus the "relative growth" of the coefficients in (1.1) for the Gottlieb polynomials stands between those of the Laguerre and Charlier polynomials but, as predicted by Theorem 8 , the distribution function is similar to that of the Charlier case, being constant except for jumps of magnitude $e^{-\lambda x}$ at $x=0,1,2, \cdots$.

\section{REFERENCES}

1. T. S. Chihara, On co-recursive orthogonal polynomials, Proc. Amer. Math. Soc. 8 (1957), 899-905.

2. D. J. Dickinson, H. O. Pollak and G. H. Wannier, On a class of polynomials orthogonal over a denumerable set, Pacific J. Math. 6 (1956), 239-247.

3. J. Favard, Sur les polynomes de Tchebicheff, C. R. Acad. Sci. Paris 200 (1935), 2052-2053.

4. M. J. Gottlieb, Concerning some polynomials orthogonal on a finite or enumerable set of points, Amer. J. Math. 60 (1938), 453-458.

5. J. Sherman, On the numerators of the convergents of the Stieltjes continued fractions, Trans. Amer. Math. Soc. 35 (1933), 64-87.

6. J. Shohat and J. Tamarkin, The problem of moments, Math. Surveys No. 1, Amer. Math. Soc. New York, 1943.

7. E. L. Stiefel, Kernel polynomials in linear algebra and their numerical applications, Nat. Bur. Standards Appl. Math. Ser. 49 (1958), 1-22.

8. T. J. Stieltjes, Recherches sur les fractions continues, Oeuvres, Tome II, Noordhoff, Groningen, 1918, pp. 402-566.

9. G. Szegö, Orthogonal polynomials, Amer. Math. Soc. Colloq. Publ. Vol. 23, Amer. Math. Soc., New York, 1939.

10. H. S. Wall, Analytic theory of continued fractions, Van Nostrand, New York, 1948.

Seattle University, Seattle, Washington 